\title{
ON THREE CASES OF ENTERECTOMY AND ENTERRORHAPHY.
}

\author{
By KENDAL FRANKS, M.D. Univ. Dubl.;
}

Fellow and Member of Council, Royal College of Surgeons, Ireland; Surgeon to the Adelaide Hospital.

[Read in the Section of Surgery, Friday, May 12, 1893.]

The abdominal cavity has for many years afforded a fruitful field for surgical enterprise. No organ contained within its walls is too awe-inspiring or too sacred to escape the sacrilegious hands of surgery. They are enclosed and protected by a membrane which was formerly regarded with reverence and dread. The peritoneum has now lost many of its te:rors. It is opened with as much sang froid as in former days it excited misgiving and fear. We have learned to treat it liumanely; to protect it against those dangers which modern science has taught us to aroid; and we have found that our confidence in it has not been misplaced. It has been shown repeatedly that every abdominal viscus can be dealt with successfully. Either entire organs or parts of organs hare been removed without injury to the organism as a whole. The hollow viscera form no exception.

It has long been known that portions of the intestine may slougl away, as a result of strangulation in hernia, and the patient recover. Sir Astley Cooper has excised a gangrenous loop twice, and Ramdohr successfully removed a piece of intestine in 1727 , and subsequently restored the continuity of the canal by a process of invagination. But on account of the inherent risks of the operation, the danger of peritonitis, the difficulties of efficient suture, and, consequently, the high mortality in the pre-antiseptic era, the operation was only attempted sporadically by the more daring spirits, 
and was not, until within the past few years, classed among the category of legitimate surgical procedures. The operation of excising a portion of the intestinal canal (enterectomy), and of suturing the divided ends together so as at once to restore the continuity of the canal (enterorrhaphy), is now very frequently performed, and with an increasing proportion of success. In this country surgery has not been behind in this respect. In $1886 \mathrm{Mr}$. M A Ardle performed pylorectomy for the first time in Ireland. It was again performed in 1889 by Sir William Stokes, and I am indebted to him for the privilege of having been present at the operation. Unfortunately both patients died. The Academy will also remember a very interesting paper by $\mathrm{Mr}$. M'Ardle, in 1887, on enterectomy and enterorrhaphy, in which he related the case of a boy, aged sixteen years, from whom he excised a piece of the ileum, about $6 \frac{1}{2}$ inches in length, half an inch from the ileo-creal valve. Enterorrhaphy was performed, and the boy made an excellent recovery. At the time of the operation the vermiform appendix was excised and closed by three Lembert sutures, and an opening in another coil of intestine, where a fistulous opening had existed between it and the diseased ileum, was freshened and sutured.

Quite recently Dr. W. J. Smyly has recorded in a paper, read before the Obstetrical Section of this Academy, the case of a woman suffering from ileo-uterine fistula. From the character of the frces, and the history of the case, it was evident that the uterus had ruptured during labour, and that a coil of small intestines had become strangulated in the rent. He says :- "Upon opening the abdomen, I found a loop of the ileum attached to the lower part of the posterior surface of the uterus. Having detached it and brought it out of the abdomen, it was found necessary to resect about two inches of the damaged portion; the divided ends were closed, and the intestinal canal restored by anastomosis, by 
means of bone plates. She made an excellent recovery, and when last heard of was working in the fields."

I am not aware of any other cases in this country in which a portion of the intestinal tract has been resected and the divided ends immediately sutured together, except three which I have performed at the Adelaide Hospital, and which I desire to lay before the Acalemy in the hope that, together with the cases to which I have referred, a nucleus may be formed, round which other cases will be gathered, forming a record which may hereafter compare favourably with the work done in intestinal surgery in other countries. The first case has already been published elsewhere, ${ }^{a}$ and I shall therefore only refer to it summarily :-

CASE I.-The patient was a man, aged fifty-eight, of very temperate habits, and with a good family history, who was admitted into hospital under my care on Oct. 19th, 1888. He had been in good health until Oct., 1887, when he had severe attacks of diarrho:t, accompanied with pain in the epigastrium, whenever he ate beef or mutton. These attacks used to last for about a day, and were followed by constipation, which continued sometimes for ten dars or a fortnight. Under medical treatment he improved, and was free from his former trouble for three months. The symptoms then returned, but the previous remedies failed to relieve him. He was obliged to restrict his diet to gruel, potatoes, bread and butter, and fish. A fortuight before $I$ saw him, one night as he was getting into bed he noticed a hard lump in the abdomen, which "seemed to fall backwards when he lay down." He was a thin man, with a sallow, cachectic aspect, suggestive of malignant lisease. On examination I found in his abdomen-which was soft and flaceid $\longrightarrow$ a very hard, nodulated tumour, about the size of at large orange, which lay in the middle line just below the umbilicus. It could be manipulated without producing pain, and was freely movable in every direction except down wards, in which lirection its range of motion seemed more limited. The diagnosis made was a tumour, probably malignant, of the omentum; but on account of the intestinal trouble I considered it was likely that the

"Trans. Medico-Chirurg. Society of London. Vol. LXXII. 1890. . 
intestine was involred, and accordingly I made preparations for dealing with the intestine previous to the operation, which took place on Oct. 30th, 1888. An incision, about 4 inches in lengtl, was made in the middle line below the umbilicus in the usual way, and the abdominal cavity was opened. The tumour, which was found lying underneath the liver, was drawn out through the wound, and proved to be a malignant growth about the size of the closed fist, growing from the hepatic half of the transverse colon, which ran through the middle of it. The omentum, which consisted merely of a connecting band between the stomach and colon, was partially infiltrated, as was also the mesocolon, between the layers of which some enlarged glands, about the size of beans, could be felt. Resection was immediately performel. The tumour, with the involved portion of intetines, being well drawn outside the abdomen, was surrounded by two large flat sponges, climped together by means of Wells' forceps, so as to shut off the field of operation as much as possible from the serous cavity beneath. The colon, on each side of the tumour, was encircled by a piece of catgut about two inches from the growtl, and each of these was tied just tight enough to occlude the bowel without interfering with its vitality. 'The colon on each side was then divided with scissors. The great omentum was ligatured bit by bit close up to the stomach above and to the colon below, and divided between them to an extent corresponding to the portion of the colon to be removed. A wedge-shaped piece of the transverse mesocolon, which was involved in the disease, was excised; all affected glands between its layers were removed. The sides of the triangular cut in the mesocolon were then approximated by means of interrupted catgut sutures, and the ends of the colon thus drawn together. The ends of the bowel were then sutured together by means of fine silk passed according to Gély's planthat is, a single row of sutures were passed parallel to the circumference of the gut, transfixing only the peritoneal and muscular coats. As each suture was passed it was knotted. When the intestinal suture was completed the colon was replaced, and the abdominal cavity was cleansed by pouring in a quatinty of boracic acid solution at blood-heat, which was allowed again to pour out, by turning the patient on his side, and the remainder was removed by careful sponging. The abdominal wound was then closed, and the patient was sent back to his bed. The operation lasted tw' hours. The length of colon remored measured five inches. On 
microscopical examination the growth proved to be a cylindrical epithelioma.

On the sixth day there was a copious evacuation from the bowels, and on the ninth day he was allowed solid food by the mouth.

It is now nearly four years and a half since the operation was performed, and the patient is still in the enjoyment of perfect heaith in the west of Ireland.

CASE II.-The second case was that of a man, aged forty-eight, who was admitted to the Adelaide Hospital on October 17, 1889, for extensive ulceration of the leg, due to varicose veins. He suffered frequently from constipation, and required large doses of medicine to affect the bowels. On December 30th, early in the morning, he was seized with violent abdominal pain about the umbilicus, and radiating from it. The next day vomiting set in and persisted. The pain came on in paroxysms, and was agonising. The bowels were absolutely confinel, neither fæces nor flatus passing after the first day. After consultation we decided on performing an exploratory laparotomy. The abdomen was opened in the usual way on January 4th, 1890. We then found that the immediate cause of his trouble was volvulus of a coil of the small intestine in the left hypochondrium. This coil for several inches was in a condition of gangrene. 'The mesentery belonging to this portion of the intestine was thick and œdematous, and we found all the veins in it completely thrombosed. They felt like cordis running through the mesentery, and evidently were not the result of recent disease. We were quite unable to account for this condition. It was evident, however, that, in spite of the serious condition of the parts, something should be attempted to relieve him of the intestinal obstruction. I accordingly determined to perform resection of the gangrenous intestine, and by immediate suture to restore the continuity of the canal. The bowel being leld firmly by the fingers of my assistant, I cut it across with a pair of strong scissors both above and below the affected portion. Sixteen inches of the gut were thus removed, together with a wedge-shaped piece of mesentery. The edges of the mesentery were first united together with a continuous catgut suture, and then the intestinal ends were brought together by means of Gély's suture passed as in the former case. The abdominal wound was closed in the ordinary manner after the "toilet" of the peritoneum 
had been accomplished. The patient recovered fairly well from the anæsthetic and from the shock of the operation. 'The next morning he informed me that, even if he did not recover, the relief he experienced was well worth the operation he had undergone. Fverything then promised well, but during the night symptoms of obstruction again appeared, and he died on January 6 th, two days after the operation. A post-mortem was made the following day by Dr. Bewley. It was then found that the lower portion of the bowel, below the line of suture, was becoming gangrenous. 'The bowel was almost impervious at the line of suture, but whether this was the result of tying the stitches too tightly, or was due to the odema accompanying the gangrene, we could not casily determine. I am inclined to think that the latter was probably the main cause, as the symptoms of obstruction did not recur for 36 hours after the suture had been applied. This was the immediate cause of death. The primary cause was due to a very remarkable and, I believe, unusual condition of thrombosis. At the autopsy we discovered that not only were the veins throughout a large piece of the mesentery completely occluded by old blood clots, but the same condition existed in the portal vein. An old firm bloodclot was found extending through its whole length, and so filled it as to leave only a minute channel beside it for the passage of the blood. We were unable to ascertain the canse or origin of this state of the veins, but to it was unquestionably due the gangrene of the gut, and the volvulus we found was, I believe, the result of this gangrene. 'There was no leakage from the site of suture.

I do not think we can justly lay the fatal result in this case at the door of resection. I think from the condition I have described it is evident that death would have resulted in spite of any treatment which might have been adopted.

CASE III.-The third case was that of a woman, aged thirty-six, who was admitted to the Adelaide Hospital on the 22 nd September, 1891. About three months previously she noticed a small swelling at the urnbilicus, which came on after lifting a heavy weight. The swelling was easily reducible into the abdomen without the least trouble or pain. She consulted a lady friend, who gave her an abdominal belt, which she had worn for a month before I saw her. During these three months she had suffered 
much from lieadache, and complained of a good deal of tenderness over the abdomen, which she thought had enlarged.

At 4 a.m. on the morning of the 22 nd of September, she awoke suddenly with a violent pain in the abdomen, and putting her hand down she discovered a large hard swelling at the umbilicus, which she tried in vain to reduce. Vomiting soon set in, and was frequent. Dr. Jones, of Rathmines, saw her about $11 \mathrm{a.m} . \mathrm{He}$ at once recognised the urgency of her case, and sent her into the Adelaide Hospital, where she arrived at 230 p.m. I saw her at 5 o'clock, and found her in the following condition :- She was a fairly well-nourished, healthy-looking woman, but she appeared to be in a very excited state. Her face was very flushed, and wore an anxious expression. She seemed to be in great pain, which she referred to the abdomen. Her tongue was furred; she did not appear to be at all collapsed. Her pulse was full, regular, and non-compressible; the temperature was normal. Her breathing was rather shallow, and she complained of its being difficult. She stated that she had had an action of the bowels the previous morning, but none since then. The urine was acid, s. g. 1030; urates were abundant, but no albumen or sugar. On examination I found that the abdomen was uniformly enlarged, and sufficiently. tense to prevent any examination of the contained organs. On percussion the flanks gave a dull note; the rest of the abdomen was tympanitic.

At the umbilicus there was a tumour about the size of an orange; it consisted of two parts-the upper ons small, with a raised rim, depressed in the centre, immediately corresponding to the umbilicus. It was of a reddish colour, evidently acutely inflamed. The lower one, larger and a little to the riglit side, was globular, extremely tense, and was of a bluish black colour. It was hard, very tender to the touch, and firmly gripped at the base. A hard, ring-like mass could be felt encircling its base. No impulse on coughing. The abdomen generally could be manipulated gently without pain, except in the immediate neighbourhood of the tumour. The patient was given a sixth of a grain of morphia at 4 p.m. and again at 11 p.m. She vomited a few times during the night, but it was not stercoraceous; she slept at intervals.

Next morning, September 23 , at 1015 a.m., $30 \frac{1}{4}$ hours after the onset of the symptoms, the patient was placed under an anrsthetic by Dr. Piel with his usual skill, and assisted by Mr. Heuston, I proceeded to operate. I made a median incision directly over the, 
tumour, and when the slin and fascia had been divided, a loop of small intestine was exposed. It was deeply congested, and at one place was quite black. Several constricting bands were successively divided, and the smaller tumour upwards and to the left was opened. It appeared like a small pouch, and contained a small portion of intestine. 'The umbiical ring was very thick, almost cartilaginous. When it was divided, the circulation gradually returned to the greater portion of the intestine, but the black portion seemed to be quite unaffected, and was evidently beginning gangrene. After a short consultation we determined to perform enterectomy-in the first place, because the intestine seemed incapable of recovery; and secondly, because the portion involved belonged to the jejunum, and an artificial anus, if established, would only postpone a fatal issue.

Accordingly $I$ proceeded first to enlarge the original opening at the umbilicus by incising the tissues downwards towards the pubes for about three inches. This was done in order to be able to draw out the intestine so as to have sufficient room for suturing. When the abdominal cavity was thus exposed, a quantity of semi-geliltinous clear fluid escaped from the wound. It was so abundant that it was impossible to proceed with the operation until the greater portion of it had been removed. I inserted my hand then through the wound to ascertain the cause, and discovered that there was a fairly large ovarian tumour, about the size of a foctus at full term; attaclied by a very broad pedicle to the broad ligament at the left side. It was a multi-locular semi-solid cyst. We determined to leave it alone for the present. We considered that the length of time necessary to resect the intestine and to suture the divided ends, together with the inherent dangers of such an operation, constituted a sufficient amount of risk in themselves without exposing the patient to the danger of prolonging the operation, and of adding to it the risks of an ovariotomy.

When a sufficient amount of the glairy fluid had been got rid of to allow us to proceed with comfort, the congested loop of intestine was drawn well forward, and then the abdominal cavity was shut off from the field of operation by means of two large flat sponges, placed one on each side of the loop of intestine, and clamped together with forceps, both above and below the loop. I determined to divide the intestine where it was healthy-that is, above and below the congested portion. Nine and a quarter inches of the gut had thus to be sacrificed. Before the intestine was divided 
the lumen of the bowel, both above and below, was occluded by means of Mr. Heuston's fingers. No clamp was employed. This method of securing the field of operation against the risks of freal contamination proved eminently convenient and satisfactory. The intestine was divided, first above and then below, with scissors, and a wedge-shaped piece was, at the same time, cut out of the mesentery, the divided vessels being secured. The noxt step in the operation was to sew up the mesentery, which was done by means of a fine catgut continuous suture. Great care was taken to close accurately the triangular spaces where the mesentery divides to enclose the gut. The method employed was this:-A suture was passed through both layers of the mesentery, close up to the intestine on one side, and then passed similarly through both mesenteric layers on the other side and firmly tied. This not only obliterated the triangular spaces, but helped to fix the divided ends of the intestine together. The remaining portions of the intestine were united by means of fine silk (Chinese twist) passed according to Gély's plan of suturing the intestines. The suture picks up only the peritoneal coat. It is passed in the following manner :$A$ long fine piece of silk is armed at each end with a fine needle. One of these needles is passed through the peritoneal coat of the upper end of the dirided intestine, about $\frac{1}{4}$ of an inch from the edge, and passing parallel to the divided edge beneath the peritoneum, emerges again at a point about $\frac{1}{4}$ of an inch from the point at which it entered. The second needle enters the peritoneum covering the lower end of the divided gut, at a point exactly opposite to the point of entrance of the first needle, and emerges at a point exactly corresponding to the point of emergence of the first needle. When the silk is drawn tight the edges of the intestine invert, and when accurately co-apted the suture is tied in a knot. The needles are then passed in again, starting from the knot, and each needle picks up another quarter of an inch of peritoneum on each side, and the suture is again tied. 'This process is repeated until the whole circumference of the bowel has been dealt with. It will thus be seen that the suture is a continuous suture, but interrupted at every point of emergence and entrance by a knot. I closed the last portion of the intestinal wound with three Lembert sutures. The parts were then thoroughly cleansed and replaced in the abdominal cavity, where they lay upon the upper portion of the ovarian tumour. As much as possible of the glairy fluid in the abdominal cavity was drained off and some of it sponged out. 
The abdominal wound was then closed, a glass drainage tube being passed down through the unper part of the wound. Sal alembroth dressings were employed. The patient did not appear at all collapsed after the operation; she had a good colour, and the pulse was excellent, when she was restored to her bed. At 1 p.m. she got, hypodermically, $\frac{1}{6} \mathrm{gr}$. of morphia, and was allowed a little ice to suck. The morphia was reneated at midnight, after which she had a good night.

I need not weary you by detailing all the particulars as to the daily treatment and progress. Suffice it to say that she was allowed one teaspoorful of white wine whey every two hours from the first day, and a zymine suppository was administered every sixth hour, and the urine was drawn off regularly. She was dressed for the first time the morning following the operation (Sept. 24th). 'The dressings were pretty we!l saturated with discharge, and by means of a syringe and indiarubber tubing $8 \frac{1}{2}$ ozs. of thick yellowish fluid were sucked up through the glass drainage tube. The next morning the dressings were again saturated, and $4 \frac{1}{2}$ ounces were sucked out. On the fourth day, as no fluid could be sucked up through the glass tube, it was removed. 'The wound healed throughout by first intention.

No food was given by the mouth exceptiag the teaspoonful of whey and a little brandy and water until the evening of the 27 th, when she got a teaspoonful of Denacyer's peptonised meat. On the 28th-that is, the sixth day-she got $\tilde{j}$ iv. of milk every four hours, and a teaspoonful of the peptonised meat every eighth hom. On Oct. 1st (the ninth day) she got some tea and toast. Nutrient enemata and zymine suppositories altemated every fourth hour during this period, and were finally abandoned on 0 ct. 5 th, the $1: 3$ th day, when she uas allowed solid food by the mouth.

During this period the patient was troubled a great deal with flatulency, but otherwise her progress left nothing to be desirel. The day following the operation slie passed some flatus, and again on the morning of the fourth day. On the fifth day she got an enema of warm water, which came away immediately with scarcely any facal matter, but the bowels moved again five hours later slightly, the motion being fluid. 'The bowels acted once or twice a day from this onward.

On the 27th of October, firc weels after the performance of the enterectomy, the patient was in such a rery satisfactory condition of lesith and strength that I considered it would be unwise 
to delay further the removal of the ovarian tumour. My chief fear was that if I delayed any adhesions which had formed would become organised and firm, and would materially increase the danger to the patient-especially as these adhesions would form between the sutured intestine and the tumour.

Accordingly, the patient being prepared in the usual manner, and placed under anisthesia, an incision was made from the umbilicus to an inch above the pubes, and when the peritoneum was opened, there was a copious discharge of the same thick, gelatinouslooking fluid as at the former operation. The tumour at once appeared. 'The largest cyst was drained through a Lawson-Tait's cannula, then the wound in the cyst-wall was enlarged sufficiently to enable me to introduce my hand, and by this means to break up the numerous eysts of which the tumour was mainly composed. At last the tumour was sufficiently reduced in size to enable me to draw it out of the abdominal cavity. There were a great number of soft adhesions, which realily yielded to the fingers. 'The tumour was attached by one broad pedicle to the broad ligament on the left side. There was also a broad strong adhesion, binding it down to the right side of the pelvis, almost constituting a second pedicle. Both of these were transfixed and ligatured in sections, and were then cut across on the side of the tumour. When it was ascertained that there were no bleeding points the abdominal carity was well flushed out with warm boracic acid solution ( 4 per cent.) and carefully sponged dry. I then sought for the sutured intestine; it was impossible to detect it with the eye, but I soon recognised it by touch. The line of union was so perfect that not even a contraction or depression on the surface marked the line of suture, but with the fingers a considerable thickening beneath the smooth surface of the peritoneum could be felt. When we had all iuspected it, the abdominal wound was closed in the usual manner. The subsequent progress requires little comment. Suffice it to say that on the 14 th day (Nor. 9th, 1891) the patient was allowed upon the sofa, and a fortnight later she left hospital perfectly recoverel. I had an opportunity of examining her six menths later. The abdomen was perfectly flaccid, soft and natural. She was in perfect health, except for occasional twinges of abdominal pain, probably due to Hatulence.

A point of great interest in this last case, and one of exceptional occurrence, was the opportunity we had of 
examining the sutured intestine in the living state five week: after enterorrhaphy had been performed. I do not think a better proof could have been afforder us of the value of this method of treatment than seeing so clearly the perfect union which nature had accomplished after suture-a union so perfect that to the eye it almost defied detection.

Another point in comnection with this case deserves comment. It is generally supposed that the method resorted to in this case-namely, resection and immediate suture in gangrenous hernia-is an ideal method, a brilliant and showy operation, but one which exposes the patient to a great and immediate risk, and therefore one to be, as a general method of practice, condemned by prudent surgeons, and discarded in favour of the older, the easier, the less brilliant, but the safer method of establishing an artificial anus. Now this is not the case. I have recently investigated the statistics of these procedures. I have collected the records of gingrenous hernia treated in various hospitals by the formation of artificial anus, and I lave also collected all the published cases of gangrenous hernia in which resection and immediate suture have been performed.

The details of this investigation will be published elsewhere, but the general result I may shortly state. The mortality which follows the formation of artificial anus, when undertaken for the relief of hernia in which the bowel is gangrenous, gives the very high percentage of $80 \cdot 7$. ()n the other hand, I have collected 222 cases in which resection and immediate suture have been resorted to, and I fint that the death-rate is only 48 per cent. Hence the more ideal operation, that which endeavour's to restore most $1 \times 1-$ fectly the slatus quo ante, is also by far the safest for tlice patient.

Time will not permit me to discuss the many interesting points which these casss as a whole suggest. I will merely 
state some conclusions to which my experience in these cases has led me:-Firstly, as to the method of suture. In all three cases I employed Gély's suture, a suture which, being continuous, is more rapidly applied than the interrupted Lembert, and I have found it answer remarkably well. I am not, however, so wedded to it as not to think that a continuous Lembert would answer at least as well. The precise method of suture which we adopt is, I believe, a matter comparatively of little importance. 'Two points, I submit, are of paramount importance. One is that we must regard that form of suture as best which, with due regard to efficiency, can be most rapidly applied. In enterectomy followed by enterorrhaphy, time is a matter of the utmost consideration. 'The other point is that we should always suture in healthy tissue. It matters very little if we remove a few inches more than is absolutely necessary. Statistics have absolutely demonstrated the truth of this, but they have also shown how fatal it is to remove one inch too little. 'Therefore, in enterectomy the best plan is to remove everything which is suspicious.

It will be observed that in my three cases I did not use a clamp to control the intestine. I relied, with confidence which was not misapplied, on the efficient control of the gut by the fingers of the gentleman who assisted me. Digital compression is far superior and much simpler than any form of clamp, and I am glad to know that so great an authority as Mr. Treves, who has himself devised a clamp, thoroughly endorses this view.

In conclusion I would observe that howerer admirable the technique. may be which we employ, however great the dexterity of the operator, failure is sure to be the reward of procrastination. If, Micawber-like, we wait to see what will turn up, patients, whose lives might ba saved by timely intervention, will assuredly drift into the rortex from which 
no tardy repentance or vain regrets on our part will avail to recall them. In conclusion I would say,-

"If it were done, when 'tis done, then 'twere well

It were done quickly."

The Crimirman said they were all of one mind in considering the communication they had just heard as one of great surgical importance. With regard to the operation of pylorectomy which Dr. Franks has alluded to, he said that the prolongation of the operation was mainly due to the sutures that were employed. He thought that if means were derised by which the intestines could be sutured more rapidly the great shock, which in many cases was the cause of death, would be avoided. He agreed with Dr. Franks in reference to the superiority of the fingers orer the clamp. because the latter must be applied with such an amount of force as could not be often properly measured. He thought too much praise could not be given to Dr. Franks for the industry he had shown in collecting the statistics of those 222 cases. An important surgical question that occurred to him was whether, in the event of finding that gangrene had taken place, they should perform resection at the site of strangulation, or whether they should perform laparotomy, and thus be enabled to see how far any infiltration had taken place. He was sure that in a large number of the cases in which the operation had been fatal, death was due to not having excised the infected portion of the gut. He thought the probabilities were that in the future they would open the abdomen and perform laparotomy, and do a much larger excision than at the seat of the gangrene. He thought too much importance must not be placed on statistics, because cases of strangulated hernia were very much more favourable than they used to be, and in some cases the gut may not have been really gangrenous at all. Where the bowel was actually gangrenous, he thought the chance of recovery would be far better by opening the abdomen and getting a full view of the seat of the lesion than by performing an operation at the seat of strangulation.

Mr. Swan remarked that the increased success of abdominal section, for all classes of visceral lesion, would cause surgeons in future to modify their treatment of strangulated hernia. He referred to a case of femoral hernia in which taxis failed and 
symptoms of acute obstruction set in. He opened the abdomen, and with the greatest ease drew the knot of intestine out of the ring, and at the same time performed the radical cure by stitching the pillars of the ring together. The case made an excellent recovery. The explanation of the facility with which the hernia was reduced was easy to demonstrate. When a hernial protrusion is pushed against a small aperture, the transverse diameter of the protrusion is increased; whereas, if the part is withdrawn from behind, there is the greatest facility for its escape. IIe thought that the cases mentioned would lead them to open the abdomen, which would be the most advantageous method in cases where there was gangrenous mischief in the gut.

Dr. BALL said that Dr. Franks had gone so very fully into this important and difficult subject that there was very little room for discussion. He saw that Mr. Lawson Tait advocated laparatomy, not alone in all cases of strangulation, but also in all cases for the radical cure of reducible hernia. He saw Mr. Tait perform this operation some years ago, and certainly the ease with which it was reduced was very remarkable.

Mr. M'ArdLe said in reference to his case of pylorectomy the delay in suturing was primarily the cause of collapse, and the operation lasted for an hour and a half. He referred to a case of Mr. 'Tait's, where after cutting down upon a femoral hernia he found it impossible to reduce the hernia, but the slightest amount of traction after doing median laparotomy resulted in drawing in the bowel with the greatest readiness. He thought, however, that when the bowel is gangrenous there is danger of infecting the peritoneum, and secondly there was danger of lacerating the bowek in their efforts at traction. He agreed with Dr. Franks as to the value of immediate resection, because they had no conception of how high the gangrene might occur. He thought septic intoxication very likely to occur from artificial anus. He considered the new anastomotic plates brought out by Dr. Murphy for suturing the intestines together much better than the plates of Senn, as too much time was spent in placing the latter plates in position. He likewise did not like to use the continuous suture, as it was likely to produce necrosed spots in the bowel. These plates had been described by Dr. Murphy as reducing the length of the operation from one and a half hours to half an hour, and in that case he thought they would be more fortunate in their pylorectomy operations. They only required to pass a strong suture round the mouth 
of the bowel and the plates fitted into position. He agreed with Dr. Franks as to the evil result of procrastination, and he was glad to see him so strongly adrocating the immediate resection and suture of the intestines.

Dr. Franks, in replying, thanked the members of the Academy for the extremely kind way in which they received his paper. With regard to Mr. Swan's treatment of strangulated femoral hernia, he himself tried that method in one case, and was surprised at the extreme ease with which the intestine came out. It also bas the adrantage that if the intestine is gangrenous you can deal easily with it. In the two cases of gangrene of the intestine which he detailed, it would be observed that in the first case he did not know that there was gangrene to deal with, and laparotomy was done as an exploratory operation. In the second case the strangulation was at the umoilicus, and this was a most favourable position for excision. He thought that in laparotomy where the hernial sac was laid open, they might leave behind a peritoneal pouch which was full of septic material, and they would be extremely likely to expose their patient to septicamia. He would prefer to do the ordinary herniotomy at the seat of gangrene, and after having cleared out the hernial sac, then open the abdomen as a secondary operation, and do resection and suture through the

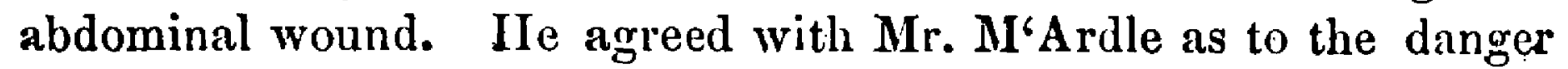
in these cases if you operate at the ring and not resect sufficient intestine. He never used Senn's plates, because he thought as much time could be saved with the continuous suture, and the plates have very often led to stricture of the intestine. With segard to the statistics, he would be entirely in accord with wilat had been said by Sir W. Stokes. However, they found that in ordinary herniotomy where the gut was healthy the mortality was 40 per cent.; he believed that the large mortality was due to the return of what looked comparatively healthy gut in to the abdomen, but which was in reality diseased. The mortality was only 48 per eent. in cases of resection for gangrenous gut, and he thought that this spoke voiumes for this operation. He said the mere percentage of deaths in cases of artificial anus was 80 per cent, whereas Mikulicz, of Warsaw, who performed 21 resections had 14 successes. He thought, therefore, that cases which die of artificial anus would be saver if resection had been performed. 